\title{
Time-Synchronization of CCSK Short Frames
}

\author{
Kassem Saied \\ Lab-STICC (UMR 6285) \\ Université Bretagne Sud, \\ Lorient, France \\ Lebanese International University, \\ Beirut, Lebanon \\ Kassem.saied@univ-ubs.fr
}

\author{
Ali Chamas Al Ghouwayel \\ Lebanese International University, \\ Beirut, Lebanon \\ EFREI, Paris, France \\ ali.ghouwayel@efrei.fr
}

\author{
Emmanuel Boutillon \\ Lab-STICC (UMR 6285) \\ Université Bretagne Sud \\ Lorient, France \\ Emmanuel.boutillon@univ-ubs.fr
}

\begin{abstract}
Efficient short Packet transmission is a key technique of the Internet of Things systems. When the data payload is small, the header used to help the receiver synchronization process becomes no longer negligible and should be shortened, and ideally removed. A Preamble-less frame generated using a non-binary error control code associated with a Cyclic Code Shift Keying (CCSK) modulation has been recently proposed. The paper presents a pragmatic approach to mitigate the time synchronization ambiguity at two levels: at symbol level thanks to an over-modulation of the CCSK symbols, and at chip level thanks to the Non-Binary code properties. Simulation results showed the efficiency of the proposed approach, where the blind frame synchronization is successfully performed at $-10 \mathrm{~dB}$.

Index Terms-Time synchronization, NB-Code properties, CCSK, Over-modulation.
\end{abstract}

\section{INTRODUCTION}

The transmission of short packets constitutes a key prerequisite for developing future Internet of Things (IoT)-based applications [1]. Usually, the frame is composed of a payload, some redundancy to correct transmission errors, and metadata known at the receiver side to help the synchronization task [2]. However, in short packet transmission, the payload is shortened and started to be comparable in size to the data used for detection and synchronization tasks. Therefore, this synchronization data cost is no longer negligible, and new efficient framing techniques are required. Moreover, Polyanskiy has demonstrated in [3], that asynchronous wireless transmission, even with short packets, does not affect the capacity of the channel. Hence, this means that classical approaches that utilize coordination between synchronization and collision avoidance are far from being optimum since the energy used for coordination is simply wasted.

In the literature, various papers investigate the frame detection and synchronization problem at low Signal to Noise Ratio (SNR) based on the transmission of a preamble for each frame [4]-[6]. This traditional preamble-based method permits high simplification of the receiver complexity, thanks to known received information. However, the employment of a preamble leads to an occupation of a significant part of the bandwidth, mainly when the message payload is small. The engineering literature has also investigated and assessed

This work has been funded by the french ANR under grant number ANR19-CE25-0013-01 (QCSP Project: https://qcsp.univ-ubs.fr/). numerous preamble-less methods for short packets [7]-[9], but all the proposed algorithms have proved their efficiency on positive decibel SNR values (i.e., SNR $>0 \mathrm{~dB}$ ).

In this paper, the authors propose to use the modulation presented in [10] to transmit short packets without any additional symbol dedicated to the function of detection and synchronization at ultra-low SNR, i.e., SNR $<0 \mathrm{~dB}$. This "preamble-less frame" is hereby referred to as a Quasi-Cyclic Short Packet (QCSP) frame. It is based on the use of a Cyclic Code Shift Keying (CCSK) modulation scheme [11] jointly associated with powerful Non-Binary (NB) forward error correction codes defined over a Galois Field $\mathrm{GF}(q)$, where $q>2$, such as NB-Low Density Parity Check (NB-LDPC) codes [12], NB-Turbo [13], NB Turbo Product Codes [14], and NB-Polar codes [15]. In this paper, we will focus only on the NB-LDPC code. The key idea is to consider the whole frame first as a preamble for detection and timing synchronization, then as an encoded payload to be decoded. The proposed detection-synchronization algorithm will process the blocks of the received samples and compute the start of the frame with high accuracy at very low SNRs, based on the efficient matching between the CCSK modulation and the NB-LDPC coding. Equally important, the QCSP frame is generated using an Over-Modulation (OM) sequence at the transmitter side to help the synchronization process after the reception of the frame without affecting the overall coding rate.

The rest of this article is organized in the following manner. In section II, the general context of our study including the system model, the detection process, and the problem statement of the synchronization are discussed. In section III, the two steps of the proposed blind time-synchronization process in a QCSP system are illustrated. Each of these steps is addressed from a theoretical perspective and validated by Monte Carlo (MC) simulation. Discussion and result analysis are given in section IV. Finally, section V concludes and gives some insights on future work.

\section{General Context of the Study}

This section illustrates the overall communication principle of the QCSP system model. It also defines the reception of the QCSP frame through an asynchronous AWGN channel. Finally, it sums up the detection process published in [16], 
states the problem of frame synchronization, and formulates the context of the proposed time-synchronization algorithm.

\section{A. Transmitter side}

Fig. 1 shows the system model of the communication link being considered, where the time-synchronization block constituting the core contribution of this work is highlighted.

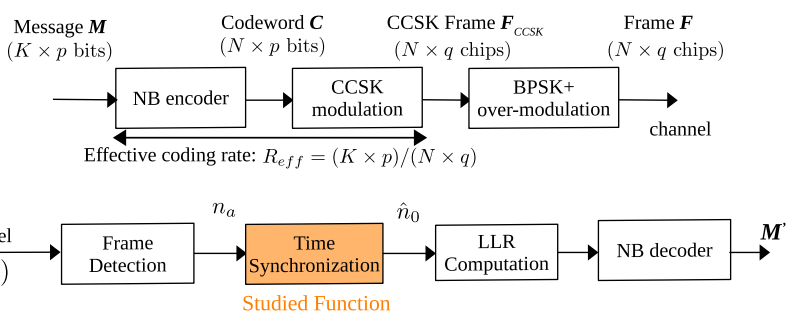

Fig. 1. Overall Communication Principle, $n_{a}$ : Time of arrival after the detection process, $\hat{n}_{0}$ : Final Chip time estimation

We consider a NB code defined over the Galois field with $q$ elements, denoted by $\operatorname{GF}(q)$. The input of the NB-code is a binary message $M$ of size $m=K \times p$ information bits, equivalently $K \mathrm{GF}(q)$ symbols. The NB-encoder generates a codeword $\boldsymbol{C}$ of $N \mathrm{GF}(q)$ symbols, i.e., $\boldsymbol{C}=$ $\left[c_{0}, c_{1}, \ldots, c_{N-1}\right]$, with $c_{k} \in \mathrm{GF}(q), k=0,1, \ldots, N-1$. Let $\boldsymbol{H}$ be the parity check matrix associated to the NB code, then the codeword $\boldsymbol{C}$ verifies $\boldsymbol{H} \boldsymbol{C}^{T}=0$, where $\boldsymbol{C}^{T}$ is the transpose of $\boldsymbol{C}$. The CCSK modulation uses a pseudorandom binary sequence $\boldsymbol{P}_{0}=\left\{P_{0}(i)\right\}_{i=0, \ldots, q-1}$ of length $q$, where $P_{0}(i) \in\{0,1\}$. The CCSK modulation, as discussed in [16], maps an element $c_{k}$ of $\mathrm{GF}(q)$ to the sequence $\boldsymbol{P}_{c_{k}}$ defined as the circular right shift of $\boldsymbol{P}_{0}$ by $c_{k}$ positions, i.e., $\boldsymbol{P}_{c_{k}}=\left\{P_{0}\left(i-c_{k} \bmod q\right)\right\}_{i=0, \ldots, q-1}$. So, the CCSK frame $\boldsymbol{F}_{C C S K}$ is thus defined as the concatenation of $N$ CCSK symbols, i.e., $\boldsymbol{F}_{C C S K}=\left[\boldsymbol{P}_{c_{0}}, \boldsymbol{P}_{c_{1}}, \ldots, \boldsymbol{P}_{c_{N-1}}\right]$. The CCSK frame used for simulation is of size $N=60$ symbols over GF(64). The associate NB-LDPC code is a regular code of coding rate $1 / 3$, with check node degree $d_{c}=3$ and variable node degree $d_{v}=2$. This NB-LDPC code is available online in [17].

In a very noisy environment, determining when the sequence starts at the exact symbol level is not a trivial task. To help the synchronization process, a symbol OM is added to the CCSK symbols. So instead of transmitting $\boldsymbol{F}_{C C S K}$ as defined before, we sent the OM QCSP frame $\boldsymbol{F}$ such that:

$$
\boldsymbol{F}=\left[(-1)^{b_{0}} \boldsymbol{P}_{c_{0}},(-1)^{b_{1}} \boldsymbol{P}_{c_{1}}, \ldots,(-1)^{b_{N-1}} \boldsymbol{P}_{c_{N-1}}\right],
$$

where $\boldsymbol{B}=\left[b_{0}, b_{1}, \ldots, b_{N-1}\right]$ with $b_{k} \in\{0,1\}$ is a sequence with good auto-correlation properties. With this additional modulation, the phase of the maximum values found at the output of the correlation block at the receiver side can be used to determine the exact window of arrival of the incoming frame. This preliminary idea will be discussed in details in section III-A.

Before transmission, the generated frame $\boldsymbol{F}$ is composed of $N \times q$ BPSK symbols, which is then shaped by a half raised cosine filter with a roll-off factor equal to 0.35 .

\section{B. Channel model}

We assume a low-cost sensor that sporadically transmits/receives small messages in an ALOHA protocol, i.e. without the prior time and frequency synchronization at the receiver side. The model of the channel is given at chip level in the sequel. The QCSP message is thus received with unknown time offset $n_{0}$ ( $n_{0}$ encompasses both the unknown times of emission, and time of propagation between the emitter and the receiver). The message is also affected by an unknown (but limited) frequency offset $f_{0}$ and an initial phase offset $\phi_{0} \in[0,2 \pi[$.

$$
y(n)=e^{j\left(2 \pi f_{0}\left(n-n_{0}\right)+\phi_{0}\right)} \boldsymbol{F}\left(n-n_{0}\right)+z(n),
$$

with $\boldsymbol{F}\left(n-n_{0}\right)=0$ when $n-n_{0} \notin \llbracket 0, N q-1 \rrbracket$. Without any prior information, $f_{0}$ and $\phi_{0}$ are supposed to be uniformly distributed in their respective interval ranges. The $z(n)$ are realizations of an Additive White Gaussian Noise (AWGN) $\mathcal{N}(\mu, \sigma)$, with mean $\mu=0$ and standard deviation $\sigma=\sqrt{\frac{10^{-\mathrm{SNR} / 10}}{2}}$, where SNR represents the signal-to-noise ratio of the transmission. The whole received frame will be denoted by $\boldsymbol{Y}$.

\section{Receiver Side}

In [16], a frame detector has been proposed at the receiver side, based on a score function $S_{n}$ indicating the detection of a newly arrived frame at a given time $n$ when a predefined threshold is attained. In [18], a novel technique named "time sliding window" allowing the computation, at low cost, the function $S_{n}$ at each index $n$ and different frequency offset hypotheses. The output of the detection process is thus an estimate of the time of arrival $n_{a}$ and a coarse estimate of the frequency offset $f_{c}$. The coarse frequency offset estimation is thus to compensate the input signal frequency offset, giving after correction, a residual frequency offset $f_{r}=f_{0}-f_{c}$. By hypothesis, $f_{r}$ will be limited to $\pm 10^{-3}$, i.e., the absolute phase offset between two chips is bounded by $2 \pi \times 10^{-3}$ radian [16]. The frequency $f_{0}$ in (2) is thus replaced by $f_{r}$ for the time synchronization task.

Let $n$ be an integer index. The correlation $L_{n}(i)$ between the vector $\boldsymbol{y}_{n}=(y(n), y(n+1), \ldots, y(n+q-1))$ and the $i^{\text {th }}$ CCSK sequence $\boldsymbol{P}_{i}$, is defined as

$$
L_{n}(i)=\sum_{l=0}^{q-1} y(n+l) P_{i}(l) .
$$

The non-coherent CCSK demodulation of the vector $\boldsymbol{y}_{n}$ consists in maximizing the absolute value of the correlation $L_{n}(i)$. Let $d_{n}$ be the hard decision of the non-coherent demodulation process, $d_{n}$ is defined as

$$
d_{n}=\underset{i=0,1, \ldots, q-1}{\operatorname{argmax}}\left\{\left|L_{n}(i)\right|\right\} .
$$

From $d_{n}$, we can define the corresponding correlation value as

$$
\gamma_{n}=L_{n}\left(d_{n}\right)
$$


and one should note that, by construction, $\left|\gamma_{n}\right|=\max \left(\left|\boldsymbol{L}_{n}\right|\right)$. Thus, we define the detection score function $S_{n}(\boldsymbol{Y})$ as

$$
S_{n}(\boldsymbol{Y})=\sum_{k=0}^{N-1} \frac{\left|\gamma_{n+k q}\right|}{\left\|\boldsymbol{y}_{n+k q}\right\|_{2}},
$$

where $\left\|\boldsymbol{y}_{u}\right\|_{2}$ represents the 2-norm value of vector $\boldsymbol{y}_{u}$.

Fig. 2 shows an example of a score function output for a frame of length $N=10$, with a CCSK modulation of length $q=64$. In this simulation, the exact time of arrival is set to $n_{0}=0$. In this curve, a AWGN channel is considered with both high $(10 \mathrm{~dB})$ and low $(-10 \mathrm{~dB}) \mathrm{SNR}$. The detection threshold is set to 20 . One can note that, in both cases, the event "a frame has arrived" is detected correctly. Nevertheless, in the case of low SNR, the maximum of the score function is not reached at time $n_{a}=0$ but at time $n_{a}=194$ chips. This time synchronization error is the sum of two events: symbol synchronization errors (here $3 \times 64$ chips), and an chip synchronization error (here 2 chips), i.e., $n_{a}=n_{0}+s q+r$, with $s=3$ and a residual chip error $r=2$.
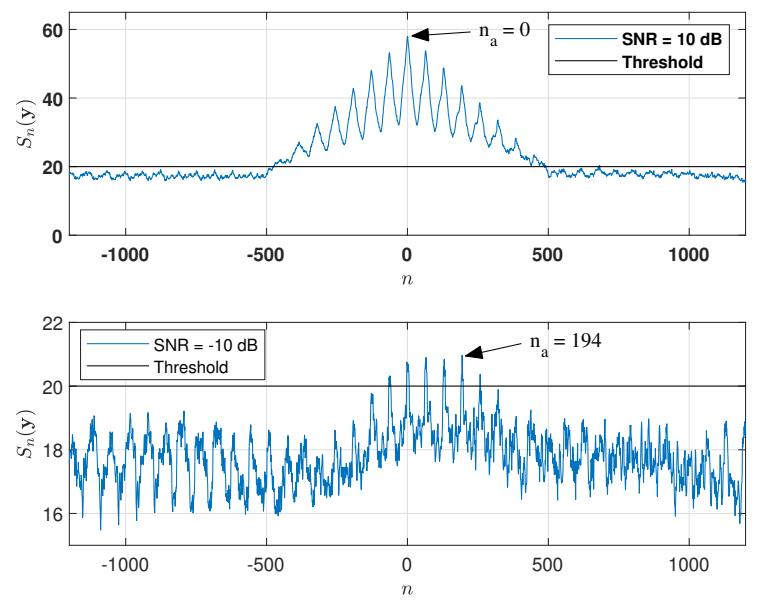

Fig. 2. Score function output for a CCSK frame with length $N=10$, $q=64$ and time offset $n_{0}=0$, considering both high $(10 \mathrm{~dB})$ and low $(-10$ dB) SNRs.

Fig. 3 shows a histogram of the chip synchronization error obtained from the time-synchronization block, after receiving 10,000 detected QCSP frames of length $N=60$ and $\boldsymbol{P}_{0}$ sequence of length $q=64$ chips. The frames are transmitted in a AWGN channel at SNR of $-10 \mathrm{~dB}$. In this simulation, more than 4,000 frames out of 10,000 are not correctly synchronized. By analyzing the probability distribution in Fig. 3 , we notice a symbol synchronization error $s$, i.e. multiple of $q$ chips, and a residual chip offset $r$, where $0 \leq s \leq 17$ and $-4 \leq r \leq 4$.

To conclude, time estimation by using the maximum of the score function alone does not give a satisfactory result at low SNR. In order to get a more accurate time synchronization process, additional side information should be used to suppress both symbol and chip time synchronization errors.

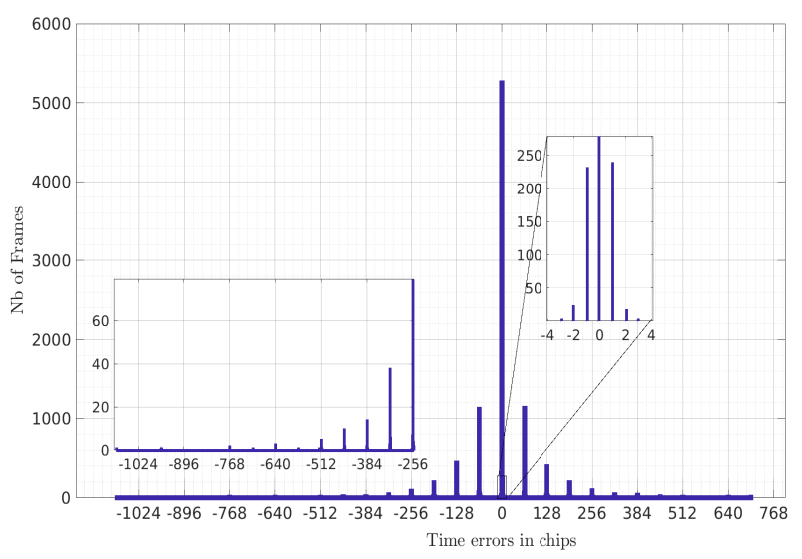

Fig. 3. Histogram of chip synchronization error with $10^{4}$ transmitted frames

\section{PRoposed Blind TIME-SYNCHRONIZATION: ILLUSTRATION AND SOME RESULTS}

This section presents in detail the two successive steps of the proposed time-Synchronization algorithm. First, an OM aided symbol synchronization process suppresses the time ambiguity at the symbol level (from $n_{a}$ to $\tilde{n}_{0}$ ). Then, a coded-aided process mitigates the last time chip ambiguity (from $\tilde{n}_{0}$ to $\hat{n}_{0}$ ), leading to the final time synchronization considered to be exact with a high probability. The input of the algorithm is a set of $2 N$ received samples that are assumed to contain a frame, as well as the coarse time estimation $n_{a}$. The output of the algorithm is the final estimation of the time of arrival $\hat{n}_{0}$.

\section{A. Symbol Synchronization: Over-Modulation}

To enhance the synchronization process at the symbol level, the OM, as defined in (1) is applied at the transmitter side. The OM generates a pre-defined phase pattern (a known sequence of $\pm 1: 1$ no phase change, and -1 ( $\pi$ rotation)) within the sequence of the symbols being transmitted. This phase pattern is expected to be recovered even when a residual frequency offset causes a rotational effect on the symbols being decoded.

Let us determine first the exact value of $\gamma_{n_{0}+k q}$ given in (5) with the hypothesis that the hard decision $d_{n_{0}+k q}$ given in (4) is correct, i.e., $d_{n_{0}+k q}=c_{k}$. In that case, according to (3).

$$
\gamma_{n_{0}+k q}=\sum_{i=0}^{q-1} y\left(n_{0}+k q+i\right) P_{c_{k}}(i) .
$$

By replacing, $y\left(n_{0}+k q+i\right)$ by its value given in (2), we have

$$
\begin{aligned}
\gamma_{n_{0}+k q} & =(-1)^{b_{k}} e^{j\left(2 \pi f_{r} k q+\phi_{0}\right)} \sum_{i=0}^{q-1} e^{j 2 \pi f_{r} i}+Z_{n_{0}+k q} \\
& =(-1)^{b_{k}} e^{j\left(2 \pi f_{r} k q+\phi_{0}\right)} \frac{1-e^{j 2 \pi f_{r} q}}{1-e^{j 2 \pi f_{r}}}+Z_{n_{0}+k q} \\
& =(-1)^{b_{k}} e^{j(\omega k+\theta)} \frac{\sin \left(\pi f_{r} q\right)}{\sin \left(\pi f_{r}\right)}+Z_{n_{0}+k q}
\end{aligned}
$$


with $\omega=2 \pi f_{r} q$ and $\theta=\phi_{0}+2 \pi f_{r}(q-1) / 2$. One can note that if $\left|f_{r} q\right| \ll 1$, then $\frac{\sin \left(\pi f_{r} q\right)}{\sin \left(\pi f_{r}\right)} \approx q$ (between 63.56 and 64 for $q=64$ and $\left|f_{r}\right| \leq 10^{-3}$ for example). Finally, $Z_{n_{0}+k q}$ represents a realization of a AWGN of zero mean and $\sqrt{q} \sigma$ as standard deviation. Let us define the $\boldsymbol{\Gamma}_{n_{0}}$ as $\boldsymbol{\Gamma}_{n_{0}}=\left(\gamma_{n_{0}}, \gamma_{n_{0}+q}, \ldots, \gamma_{n_{0}+q(N-1)}\right)$. In the absence of wrong decision, the term by term vector multiplication of $\Gamma_{n_{0}}$ and $\boldsymbol{B}$ gives

$$
\boldsymbol{\Gamma}_{n_{0}} \odot \boldsymbol{B} \approx q e^{j \theta}\left(1, e^{j \omega}, e^{j 2 \omega}, \ldots, e^{j(N-1) \omega}\right)+\boldsymbol{Z}_{n_{0}},
$$

with $\boldsymbol{Z}_{n_{0}}$ a vector of $N$ AWGN samples of zero mean and $\sqrt{q} \sigma$ standard deviation. In summary, in the absence of wrong decision, $\boldsymbol{\Gamma}_{n_{0}} \odot \boldsymbol{B}$ is a pure sinusoidal vector of length $N$ and frequency $\omega$ affected by AWGN. This property is used to suppress time ambiguity at the symbol level. In fact, the initial time estimation $n_{a}$ of $n_{0}$ is affected by $s$ symbol errors and $r$ chip errors. By neglecting the chip errors in this first step of the algorithm, we get $n_{0}=n_{a}+s q$. If $s \neq 0$, the vector $\boldsymbol{\Gamma}_{n_{a}} \odot \boldsymbol{B}$ does not generate a pure sinusoidal of length $N$, but will include a sequence of $N-s$ successive components of $\left(e^{j k \omega}(-1)^{b_{k}}(-1)^{b_{k-s}}\right)_{k \in \llbracket \max (0, s), \min (N, N+s) \rrbracket}$ that contains no regular pattern thanks to the choice of $\boldsymbol{B}$. It is thus possible to estimate the value of $s$ by selecting the value $\tilde{s}$ that makes the hypothesis " $\boldsymbol{\Gamma}_{n_{a}+s q} \odot \boldsymbol{B}$ is a pure sinusoidal affected by noise" the more likely. This estimated value $\tilde{s}$ will give an updated version of the estimation of the arrival time $n_{a}: \tilde{n}_{0}=n_{a}+\tilde{s} q$.

Our first attempt was then to select the value $s$ that maximizes the maximum module of the Fast Fourier Transform of the $\boldsymbol{\Gamma}_{n_{a}}(s) \odot \boldsymbol{B}$ vector. In other words, the ambiguity on symbol position is solved by taking $\tilde{s}$ as

$$
\tilde{s}=\underset{s \in \llbracket-N / 2, N / 2 \rrbracket}{\operatorname{argmax}}\left\{\max \left\{\left|\mathrm{FFT}\left(\boldsymbol{\Gamma}_{n_{a}+s q} \odot \boldsymbol{B}\right)\right|\right\}\right\} .
$$

This method is simple and suppresses the symbol ambiguity in the large majority of the cases. Nevertheless, when decisions on several symbols are wrong, the phase of the associated symbols is also wrong, making the proposed method prone to some synchronization errors. To mitigate this problem, we propose to weight the values of $\gamma_{s}$ by a coefficient that indicates the reliability of the decision. Let $d_{n}^{2}$ be the index of the second-highest decision in (3), and $\epsilon_{n}=L_{n}\left(d_{n}^{2}\right)$ i.e.,

$$
d_{n}^{2}=\underset{i=0,1, \ldots, q-1, i \neq d_{n}}{\operatorname{argmax}}\left\{\left|L_{n}(i)\right|\right\} .
$$

The relative ratio $\alpha_{n}$ between $\left|\gamma_{n}\right|$ and $\left|\epsilon_{n}\right|$ defined as $\alpha_{n}=$ $\frac{\left|\gamma_{n}\right|-\left|\epsilon_{n}\right|}{\left|\gamma_{n}\right|}$ is a good indicator of the reliability of the decision. For example, $\alpha_{n}=0$ means that the decisions $d_{n}$ and $d_{n}^{2}$ have same reliability, thus $d_{n}$ is not a reliable decision. On the contrary, $\alpha_{n}$ close to one indicates a very reliable decision. Let $A_{n}$ be the vector $A_{n}=\left(\alpha_{n}, \alpha_{n+q}, \ldots, \alpha_{n+(N-1) q}\right)$, the Weighted OM (WOM) algorithm is thus given as:

$$
\tilde{s}=\underset{s \in \llbracket-N / 2, N / 2 \rrbracket}{\operatorname{argmax}}\left\{\max \left\{\left|\mathrm{FFT}\left(A_{n_{a}+s q} \odot \boldsymbol{\Gamma}_{n_{a}+s q} \odot \boldsymbol{B}\right)\right|\right\}\right\}
$$

Fig. 4 shows the value of $\max \left\{\left|\operatorname{FFT}\left(A_{n_{a}+s q} \odot \boldsymbol{\Gamma}_{n_{a}+s q} \odot \boldsymbol{B}\right)\right|\right.$, where four received frames are considered with $N=64$, $q=64$ at SNR of $-10 \mathrm{~dB}$. The initial coarse time estimations $n_{a}$ given by the detection algorithm for the four frames are affected by a synchronization time error $s \times q$ with $s=-9,0,2$ and 7 respectively. In each case, (12) allows a correct estimation of $s$.

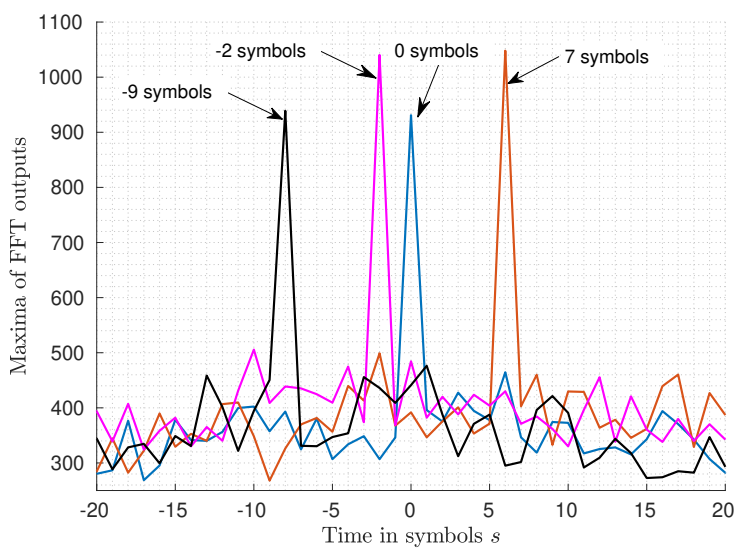

Fig. 4. Illustration of equation (12) over 4 different received frames, $N=$ $60, q=64$, at $-10 \mathrm{~dB}$.

Table I shows the probability distribution of chip synchronization error $\tilde{e}=n_{0}-\tilde{n}_{0}$ over $10^{4}$ frames. The first observation is that all the frames are successfully synchronized at the symbol level thanks to the first processing step. At this end of the synchronization process, more than $90 \%$ of the frames are correctly synchronized in time. The remaining $10 \%$ suffers from a low amplitude error synchronization, with $\tilde{e} \in[-4,4]$. As mentioned before, these chip offsets will be solved with the help of the parity checks of the NB-LDPC decoder. This step will be presented and discussed in detail in the next step of the synchronization process.

TABLE I

NUMBER OF FRAMES WITH CHIP ERROR ẽ OUT OF $10^{4}$ FRAMES.

\begin{tabular}{|c|c|c|c|c|c|c|c|c|c|}
\hline$\tilde{e}$ & -4 & -3 & -2 & -1 & 0 & 1 & 2 & 3 & 4 \\
\hline nb of frames & 2 & 7 & 50 & 440 & 9021 & 420 & 47 & 12 & 1 \\
\hline
\end{tabular}

To conclude, two hypotheses have been assumed to justify the mathematical model used to determine the best estimation $\tilde{s}$ of the parameter $s$. The first hypothesis was the fact that $n_{0}=n_{a}+s q$, while in fact $n_{0}=n_{a}+s q+r$, with $r$ having the distribution given in table I. The second hypothesis is not explicitly formulated but, due to the channel noise, not all the values of $d_{n_{0}+k q}$, are correctly estimated, leading to values of $\gamma_{n_{0}+k q}$ different than the expression given in (5). Nevertheless, MC simulations show that, even if the hypothesis used to justify the mathematical model is not fully exact, the method remains efficient in practice.

\section{B. Coded aided fine chip synchronization}

The objective of the chip synchronization is to estimate the remaining chip errors $r=n_{0}-\tilde{n}_{0}$. To do so, we first adopt 
the Syndrome-Based (SB) time synchronization presented in [19] [20] to the proposed QCSP frame. Then, we improve this method by replacing the hard metric, which is the check node syndrome calculation, with a soft Variable Node-Based (VNB) metric.

Let us first describe some features of the NB-LDPC code defined by the parity check matrix $\boldsymbol{H}$ of $N-K$ rows and $N$ columns. The code is assumed to be regular, with weights $d_{v}=2$ columns and $d_{c}=3$ rows (code rate $1-2 / 3=1 / 3$ ). Let $\mathcal{M}(j)$ be the set of the $d_{c}=3$ non-null positions of the $j^{\text {th }}$ row of $\boldsymbol{H}$. The $j^{\text {th }}$ parity check equation for a vector $\boldsymbol{X}=(x(0), x(1), \ldots, x(N-1))$ is defined as

$$
\sum_{i \in \mathcal{M}(j)} h(j, i) x(i)=0
$$

Let us define $\boldsymbol{D}_{n}=\left(d_{n}, d_{n+q}, \ldots, d_{n+(N-1) q}\right)$. In the absence of decoding error, $\boldsymbol{D}_{n_{0}}$ is a codeword and thus, all the parity checks are fulfilled. If the number of detection errors is low enough, only a few parity checks will not be fulfilled. On the other side, if $n \neq n_{0}, \boldsymbol{D}_{n}$ can be seen as a random vector, and thus, the number of non-verified parity checks will be in average close to $N-K$ since each check node has 1 chance over $q$ to be fulfilled. Let us define $\operatorname{NoZ}\left(D_{n}\right)$ (Number of Zero) the function that counts the number of satisfied parity checks. The syndrome-aided chip synchronization, over multi hypotheses $\left.r \in \llbracket-\frac{q}{8}, \frac{q}{8} \rrbracket\right)$, is given as

$$
\hat{r}=\underset{r \in \llbracket-\frac{q}{8}, \frac{q}{8} \rrbracket}{\operatorname{argmax}}\left\{\operatorname{NoZ}\left(\boldsymbol{D}_{\tilde{n}_{0}+r}\right)\right\} .
$$

This method is efficient as long as the number of decoding errors is low enough. Nevertheless, it is not necessarily always the case and thus this method fails sometimes. To mitigate this problem, we propose to replace the SB method seen at the check node level with a VNB method treating a "soft syndrome" seen at the variable node level. The idea is to perform one decoding iteration of the code with the hard decision vector $\boldsymbol{D}_{n}$. This decoding iteration generates $d_{v}=2$ check to variable messages for each variable. The two messages $M_{j \rightarrow i}$ sent by checks $j \in \mathcal{N}(i)$ to variable $i$ message is defined as in [21],

$$
M_{j \rightarrow i}\left(\boldsymbol{D}_{n}\right)=h(j, i)^{-1} \sum_{i^{\prime} \in \mathcal{M}(j), i^{\prime} \neq i} h\left(j, i^{\prime}\right) D_{n}\left(i^{\prime}\right) .
$$

Let us defined $\boldsymbol{D}_{n}^{2}$ as $\boldsymbol{D}_{n}^{2}=\left(d_{n}^{2}, d_{n+q}^{2}, \ldots, d_{n+(N-1) q}^{2}\right)$. Similarly, $\boldsymbol{D}^{3}$ is defined as the decision vector of the third-highest reliable decision, i.e., $D^{3}=$ $\left(d_{n}^{3}, d_{n+q}^{3}, \ldots, d_{n+(N-1) q}^{3}\right)$ with $d_{n}^{3}$ defined as

$$
d_{n}^{3}=\underset{i=0,1, \ldots, q-1, i \neq d_{n}, d_{n}^{2}}{\operatorname{argmax}}\left\{\left|L_{n}(i)\right|\right\} .
$$

Let us define the score function $G_{n}$ as:

$$
G_{n}=\sum_{i=0}^{N-1} \sum_{j \in \mathcal{N}(i)} f\left(M_{j \rightarrow i}\left(\boldsymbol{D}_{n}\right), D_{n}(i), D_{n}^{2}(i), D_{n}^{3}(i)\right),
$$

where $f\left(m, x, x_{2}, x_{3}\right)$ is a function of $\mathrm{GF}(\mathrm{q})^{4}$ to the real number that associate a value 1 if $m=x, 0.9$ if $m=x_{2}$, 0.8 if $m=x_{3}$, and 0 otherwise.

The new proposed method is thus:

$$
\hat{r}=\underset{r \in \llbracket-\frac{q}{8}, \frac{q}{8} \rrbracket}{\operatorname{argmax}}\left\{G_{\tilde{n}_{0}+r}\right\},
$$

The addition of the values $1,0.9,0.8$, and 0 corresponds to the following analysis. In case of correct synchronization (i.e., correct hypothesis $r$ giving $n_{0}=\tilde{n}_{0}+r$ without any decision error in $\boldsymbol{D}_{n_{0}}$, the output of the check nodes $M_{j \rightarrow i}\left(\boldsymbol{D}_{n_{0}}\right)$ will equal $D_{n_{0}}(i)$. If $\boldsymbol{D}_{n_{0}}$ contains some errors, most probably the outputs of parity checks may have some elements in $D_{n_{0}}^{2}$, and less probable in $D_{n_{0}}^{3}$. In worst-case scenarios, the output is neither element of the three different vectors. It is worth noticing here that in the case of good synchronization and with no errors on the first decisions $\boldsymbol{D}_{n_{0}}$, the output of the score function $G_{n_{0}}$ will be, $G_{n_{0}}=N \times 2$ (since $d_{v}=2$ ). Note that this method is simple and efficient, and more elaborated methods can also be organized as future work.

Fig. 5 illustrates the use of equation (18) by applying it over 4 independent received QCSP frames affected by the chips errors $-3,0,0$ and 3, respectively. Then, in each of the cases, (18) solve the problem.

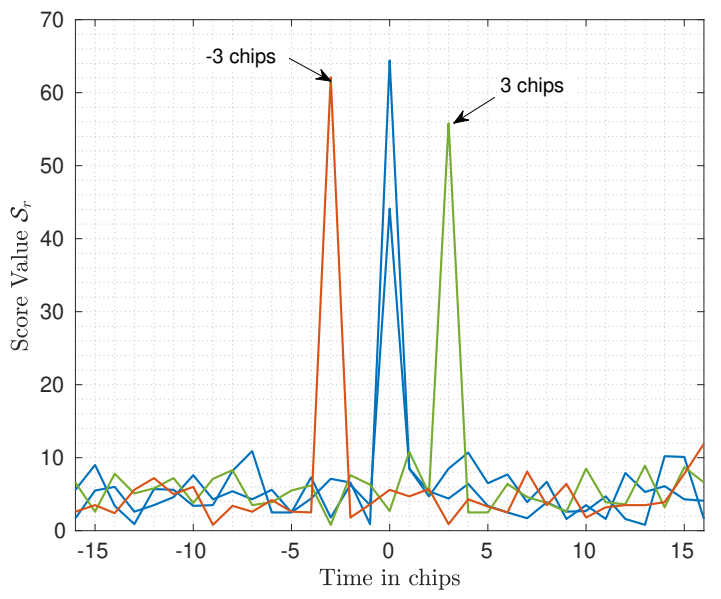

Fig. 5. Examples of $G_{\tilde{n}_{0}+r}$ values (see (18) as a function of $r$.

After this chip synchronization process, we found out that, at $\mathrm{SNR}=-10.25$, all the $10^{4}$ received QCSP frames have been perfectly synchronized.

\section{Simulation Results}

This section presents the simulation results of the QCSP receiver implemented using the different combinations of the symbol synchronization methods (OM, WOM) and the chip synchronization methods (SB, VNB). The MC simulations are run over a AWGN channel with stopping criteria of 100 miss-detected or miss-synchronized frames, NB-LDPC encoder with coding rate $R_{c}=1 / 3, q=64$, and time 
and frequency shifts considered to be uniform randomlydistributed. The blue curve in Fig. 6 shows the miss-detection probability $\mathcal{P}_{\text {md }}$ of the blind detection method as presented in [16], where the time and frequency bins being considered are $q / 4$ and $\pi / 2$, with a probability of false alarm $P_{f a}=10^{-6}$. The second step is to feed the blind-synchronization block with the frames being successfully detected and then assess the synchronization performance. Note that the frames being processed contain a residual frequency error bounded by $\pm 10^{-3}$ [16]. The solid red curve shows the probability of misssynchronization $\mathcal{P}_{\mathrm{ms}}$ obtained using the WOM-VNB method, where a gain of $0.25 \mathrm{~dB}$ is obtained with respect to the dashed red curve representing the OM-VNB case. This gain shows the impact of the weighting technique in the $\mathrm{OM}$ method. When comparing the WOM-VNB (solid red curve) to WOM$\mathrm{SB}$ (green curve), a gain of $0.5 \mathrm{~dB}$ is noticed which shows the efficiency of the VNB technique as compared to the SB one. To the best of our knowledge, this is the first blindsynchronization algorithm that reaches a $\mathcal{P}_{\mathrm{ms}}$ of the order of $10^{-5}$ at very low SNR $(-10 \mathrm{~dB})$.



Fig. 6. $\quad \mathcal{P}_{\mathrm{md}}$ and $\mathcal{P}_{\mathrm{ms}}$ vs $\mathrm{SNR}$ for the QCSP receiver using several combinations of the symbol and chip synchronization methods.

\section{CONCLUSION}

The paper presented a preamble-less time-synchronization algorithm for QCSP frames operating at very low SNR $(-10$ $\mathrm{dB}$ ) with a probability of miss-synchronization of the order of $10^{-5}$. The proposed algorithm mitigates the time ambiguity in two consecutive steps. The first step is at the symbol level where an OM technique has been proposed, and then enhanced with a weighted version privileging the symbols received with high reliability. The second step is at the chip level by taking advantage of the NB-LDPC code structure.

This work will be extended in several directions in the near future. First, performance assessment at different frame lengths, code rates, and $\operatorname{GF}(q)$ will be addressed, along with the mitigation of the residual frequency errors. Then, the different scenario of simulations considered over a AWGN channel will be run over the Rayleigh fading channel.

\section{REFERENCES}

[1] K. Mekki, E. Bajic, F. Chaxel, and F. Meyer, "A comparative study of LPWAN technologies for large-scale IoT deployment," vol. 5, pp. 1-7, March 2019.

[2] H. Rahbari and M. Krunz, "Exploiting Frame Preamble Waveforms to Support New Physical-Layer Functions in OFDM-Based 802.11 Systems," IEEE Transactions on Wireless Communications, vol. 16 , no. 6, pp. 3775-3786, 2017.

[3] Y. Polyanskiy, "Asynchronous Communication: Exact Synchronization, Universality, and Dispersion," IEEE Transactions on Information Theory, vol. 59, no. 3, pp. 1256-1270, March 2013.

[4] S. Kim, K. Joo, and Y. Lim, "A delay-robust random access preamble detection algorithm for LTE system," in 2012 IEEE Radio and Wireless Symposium, 2012, pp. 75-78

[5] Z. Ye, C. Duan, P. V. Orlik, J. Zhang, and A. A. Abouzeid, "A Synchronization Design for UWB-Based Wireless Multimedia Systems," IEEE Transactions on Broadcasting, vol. 56, no. 2, pp. 211-225, 2010.

[6] M. Schlüter, M. Dörpinghaus, and G. P. Fettweis, "Bounds on Phase, Frequency, and Timing Synchronization in Fully Digital Receivers With 1-bit Quantization and Oversampling," IEEE Transactions on Communications, vol. 68, no. 10, pp. 6499-6513, 2020.

[7] A. Azari, P. Popovski, G. Miao, and C. Stefanovic, "Grant-Free Radio Access for Short-Packet Communications over 5G Networks," in GLOBECOM 2017 - 2017 IEEE Global Communications Conference, 2017, pp. 1-7.

[8] B. Bloessl and F. Dressler, "mSync: Physical Layer Frame Synchronization Without Preamble Symbols," IEEE Transactions on Mobile Computing, vol. PP, pp. 1-1, 022018.

[9] P. Walk, P. Jung, B. Hassibi, and H. Jafarkhani, "MOCZ for Blind Short-Packet Communication: Practical Aspects," IEEE Transactions on Wireless Communications, vol. 19, no. 10, pp. 6675-6692, 2020.

[10] O. Abassi, L. Conde-Canencia, M. Mansour, and E. Boutillon, "NonBinary Low-Density Parity-Check coded Cyclic Code-Shift Keying," in 2013 IEEE Wireless Communications and Networking Conference (WCNC), April 2013, pp. 3890-3894.

[11] G. M. Dillard, M. Reuter, J. Zeiddler, and B. Zeidler, "Cyclic code shift keying: a low probability of intercept communication technique," IEEE Transactions on Aerospace and Electronic Systems, vol. 39, no. 3, pp. 786-798, July 2003.

[12] O. Abassi, L. Conde-Canencia, M. Mansour, and E. Boutillon, "Nonbinary coded CCSK and Frequency-Domain Equalization with simplified LLR generation," in 2013 IEEE 24th Annual International Symposium on Personal, Indoor, and Mobile Radio Communications (PIMRC), Sep. 2013, pp. 1478-1483.

[13] H. le Blevec, R. Klaimi, S. Weithoffer, C. Abdel Nour and A. Baghdadi, "Low complexity non-binary turbo decoding based on the local-SOVA algorithm," in the IEEE international conference on Topics in Coding (ISTC'2021), Sept. 2021, pp. 1-6.

[14] R. Klaimi, C. A. Nour, C. Douillard, and J. Farah, "Low-complexity decoders for non-binary turbo codes," in 2018 IEEE 10th International Symposium on Turbo Codes Iterative Information Processing (ISTC), Dec 2018, pp. 1-5.

[15] V. Savin, "Non-Binary Polar Codes for Spread-Spectrum Modulations," in the IEEE international conference on Topics in Coding (ISTC'2021), Sept. 2021, pp. 1-6.

[16] K. Saied, A. C. A. Ghouwayel, and E. Boutillon, "Quasi Cyclic Short Packet for asynchronous preamble-less transmission in very low SNRs," Preprint HAL, no. 02884668, 2020.

[17] (2020) Web site on Non-Binary LDPC. [Online]. Available: http://wwwlabsticc.univ-ubs.fr/nb-ldpc/.

[18] C. Monière, K. Saied, B. Legal, and E. Boutillon, "Time sliding window for the detection of CCSK frames," in the IEEE Workshop on Signal Processing Systems (SiPS'2021), Oct. 2021, pp. 1-6.

[19] R. Imad and S. Houcke, "Blind frame synchronization and phase offset estimation for coded systems," in 2008 IEEE 9th Workshop on Signal Processing Advances in Wireless Communications, 2008, pp. 11-15.

[20] R. Imad, C. Poulliat, and S. Houcke, "Frame Synchronization Techniques for Non-Binary LDPC Codes over GF(q)," 01 2011, pp. $1-6$.

[21] X. Zhang, F. Cai, and S. Lin, "Low-complexity reliability-based message-passing decoder architectures for non-binary ldpc codes," IEEE Transactions on Very Large Scale Integration (VLSI) Systems, vol. 20, no. 11, pp. 1938-1950, 2012. 